\title{
IMPACT OF NUMBER OF FIBROMYALGIA TENDER POINTS ON DISEASE ACTIVITY IN SYSTEMIC LUPUS ERYTHEMATOSUS PATIENTS
}

\author{
CHOWDHURY SM ${ }^{1}$, CHOWDHURY MHK ${ }^{2}$, HOSSAIN SZ ${ }^{3}$, RANA M ${ }^{4}$, HOSSAIN MZ ${ }^{5}$, YESMIN S6
}

\begin{abstract}
Background: Fibromyalgia tender points (FMTP) have consistently reported higher number count in systemic lupus erythematosus (SLE) patients compared with the general population.

Objective: The purpose of this study was to determine the association and impact of fibromyalgia tender points on disease activity in SLE patients.

Methods: Sixty seven female SLE patients and equal number of asymptomatic, age and sex matched control subjects were enrolled in this observational study. The study was performed between April 2005 and October 2006. After fulfilling the certain inclusion criteria, obtained data were recorded in a structured questionnaire. 18 FMTP sites and 6 control sites were examined by standardized manual tender point survey system. Systemic lupus erythematosus disease activity index (SLEDAI) was used covering all 9 organs and 24 descriptors with pre assigned severity weights. The scores ranged from $O$ ( no disease activity) to 105 (maximum disease activity). Both the SLE cases and the control group were classified into two subgroups namely '0-10 FMTP' having 10 or less FMTP and >11 FMTP' bearing 11 or more tender points.

Results: The age (mean $\pm S D$ ) of the SLE patients and controls were $26.82 \pm 8.02$ and 29.67 \pm 10.80 years respectively. No significant difference in age $(p=0.091)$ and in family income $(p=$ $0.065)$ was observed but significant difference was observed in level of education ( $p=0.009$ ) and in occupation ( $p=0.004)$. At enrolment, the number of FMTP (mean $\pm S D$ ) in SLE patients and control groups were $8.80 \pm 6.40$ and $3.63 \pm 4.90$ respectively. The difference was significant $(p<0.001)$. The SLEDAI score (mean $\pm S D$ ) in SLE patients was 11.75 \pm 9.85 . Further, the SLEDAI score of 'O-10 FMTP' and >11FMTP' subgroups of SLE were 8.49 17.34 and $17.58 \pm 11.17$ respectively. The difference was again significant $(p<0.001)$. Hence the disease activity was positively correlated ( $r=+0.439, p<0.001)$ with FMTPs in SLE patients. Meanwhile, no significant correlation was observed between disease duration $(r=+0.085, p=0.495)$ and SLEDAI and similarly no significant correlation was also observed in disease duration with FMTP; $0-10$ and $>11$ FMTP groups of SLE ( $p=0.605)$

Conclusions: SLE patients with higher number of FMTPS are significantly associated with increased scores of SLEDAI. Hence, it can be said that there is a linear correlation between the number of FMTPS and the disease activity in SLE patients. Interestingly, no significant correlation was observed between 'disease duration and SLEDAI' as well as between 'disease duration and FMTP'.
\end{abstract}

Key words: Fibromyalgia tender points (FMTP), Systemic lupus erythematosus (SLE), Systemic lupus erythematosus disease activity index (SLEDAI)

J Dhaka Med Coll. 2015; 24(2) : 99-107.

\section{Introduction}

Fibromyalgia (FM) is a nonarticular rheumatologic condition with diverse set of signs and symptoms, including diffuse and chronic widespread musculoskeletal pain, multiple regional tender points (TP) on examination, poor and nonrestrictive sleep, stiffness, and fatigue ${ }^{1,2}$. Diagnosis of FM is made of using 1990 American College of Rheumatology (ACR) diagnostic criteria ${ }^{1}$. FM

1. Dr. Salauddin Mamun Chowdhury, Department of Medicine, 250 Bed General Hospital, Noakhali.

2. Dr. Mohiuddin Humayun Kabir Chowdhury, Department of Medicine, 250 Bed General Hospital, Noakhali.

3. Dr. Syed Zakir Hossain, Department of Medicine, Dhaka Medical College Hospital, Dhaka.

4. Dr. Masud Rana, Department of Medicine, Abdul Malek Ukil Medical College Hospital, Noakhali.

5. Dr. Mohammad Zaid Hossain, Associate Professor, Department of Medicine, Dhaka Medical College, Dhaka 6. Dr. Sabrina Yesmin, Phase-B Resident, Department of Rheumatology, BSMMU, Dhaka

Correspondence : Dr. SM Chowdhury, Department of Medicine, 250 Bed General Hospital, Noakhali. Email: drsalauddin_mamun@yahoo.com 
was cited as Chronic widespread musculoskeletal pain 3 months or more, involving three or more segment of the body or presence of 11 or more out of 18 specific tender point sites on digital palpation with an approximate force of $4 \mathrm{~kg} / \mathrm{cm}^{2}$ or pressure which whitens the nail bed of thumb ${ }^{2,3}$. Widespread pain was defined in five regions: the right side of body, the left side of body, the upper part above the waist, the lower part below the waist and the axial skeleton, e.g, cervical spine, anterior chest, thoracic spine or low back ${ }^{4}$.

The prevalence of FM ranges between 0.75 and 3 percent in the general population ${ }^{6}$. In Bangladesh, the point prevalence of FM is about 4.3 percent according to a COPCORD study ${ }^{7}$. Extrapolation of prevalence rate of SLE in Bangladesh is 0.54 percent $^{7}$. Distinguishing FM in the setting of SLE is difficult because of similarity of some of the symptoms of the two diseases. Symptoms of SLE are attributed to activation of the immune system, whereas the vast majority of FM patients show no evidence of autoimmune activity ${ }^{6}$.

Since the 1950s there have been at least 60 attempts at developing a disease activity index in SLE patients. An assessment of disease activity, severity, a damage index and quality of life measures is essential for treatment decision, as well as important in assessing response to treatment. The current commonly used indices are the BILAG (British Isles Lupus Assessment Group), SLAM (Systemic Lupus Activity Measure), ECLAM (European Community Lupus Activity Measure) and the SLEDAI (Systemic Lupus Erythematosus Disease Activity Index) ${ }^{8}$. Among these, SLEDAI is most widely used in routine clinical practice and shown to be reliable and reproducible in different countries ${ }^{9}$. The SLEDAI was developed in 1985 at the 'Conference in Prognosis Studies in Lupus' a convention held in Toronto ${ }^{10}$. It represents the consensus of a group of experts in the field of lupus research.

The SLEDAI scale has 24 items with a maximum score of 105 . The index measures activity in the preceding 10 Days. The items are scored as being present or absent. The SLEDAI is a reliable validated model of global assessment of disease activity, and it generated a 'weighted' index of 9 organ systems, 24 descriptors for disease activity in SLE. The SLEDAI scorings are as follows: 8 for central nervous systems and vascular, 4 for renal and musculoskeletal, 2 for serosal, dermal, immunologic and 1 for constitutional and haematologic. The maximum theoretical score is 105 (maximum disease activity) and minimum is 0 (no disease activity), but in practice, few patients had scores greater than $45^{9}$. A score of 6 is clinically important and influences the majority of physicians to treat ${ }^{11}$.

Gladman et al. (1997) ${ }^{12}$ showed that FM did not correlate to the total score or to any of the components of SLEDAI. As well, when SLEDAI descriptors were grouped into 9 organ systems, no relationship was found too. Gladman et al. (1994), in a separate study also found that FM, as assessed by the tender point count, correlated with all domains of SF-36 (short form 36 , a health status measuring tool) but again not with the SLEDAI ${ }^{8}$.

The research question of this study was to assess if the number of FMTPs correlates with disease activity in SLE patients.

\section{Methods}

Study Sample

Total sixty-seven female SLE patients and equal number of asymptomatic age and sexmatched female subjects were enrolled in this observational study as control on the basis of a set of inclusion and exclusion criteria. The samples were taken by recruiting SLE patients attending the SLE Clinic of Rheumatology Wing, BSMMU. The study period was from April 2005 to October 2006. All the patients and the controls were requested to give informed consent to participate and they did the same accordingly. After fulfilling the inclusion criteria, a detailed history was taken and thorough physical examinations were performed to record the obtained data in a structured questionnaire. The questionnaire was designed to get sociodemographic variables like age, occupation, house-hold income, level of education, disease duration and clinical information, such as fibromyalgia tender points' number count, SLEDAI score and clinical features of SLE in terms of ACR criteria to diagnose SLE.

\section{Fibromyalgia tender point survey}

All SLE patents and controls were further requested to denote the presence or experience of persistent pain area at any body part. The 
subjects were subsequently examined to determine the number of fibromyalgia tender points by digital palpation with the thumb-pad of dominant hand i.e. the force was so in such strength that just whitened the nail bed to the site examined. Eighteen active sites (9 pairs) were examined: at sub occipital muscle insertion, anterior aspects of intertransverse spaces at C5-C7, midpoint of the upper border of trapezius, supraspinatus at origin above the scapular spine, second rib at the second costochondral junction, at lateral epicondyle 2 $\mathrm{cm}$ distally, upper and outer quadrant of buttock, greater trochanter just posterior to the trochanteric prominence and knee at the medial pad of fat proximal to the joint line. Six control sites ( 3 pairs) were examined. Forearm: at distal third of the forearm, thumbnail and Midfoot: at the midfoot of the dorsal third metatarsal (Fig.-1) 13. The following scoring system for grading the severity of tender points (18) was used: $0=$ no pain; 1 (mild) = complaint

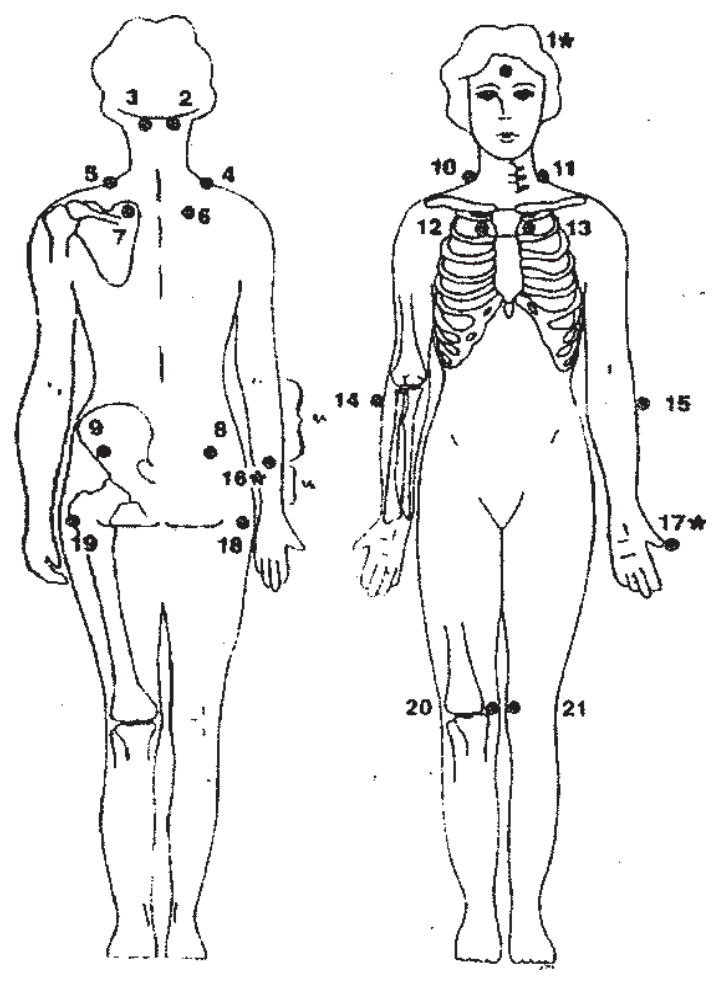

The MTPS scoring form (after Okufuji et al., 1997)

\section{Survey and control* ${ }^{*}$ ites}

\begin{tabular}{|c|c|c|}
\hline Seated & Right & Left \\
\hline Mid-forehead $(*)$ & \multicolumn{2}{|c|}{$1 \ldots \ldots . . *^{*}$} \\
\hline Occiput: suboccipital muscle insertions & $2 \ldots \ldots \ldots$ & $3 \ldots \ldots$. \\
\hline Trapezius: midpoint of upper border & $4 \ldots \ldots .$. & $5 \ldots . .$. \\
\hline Supraspinatus: above medial border of scapular spine & $6 \ldots \ldots . .$. & $7 \ldots \ldots .$. \\
\hline Gluteal: upper outer quadrant of buttocks & $8 \ldots \ldots .$. & $9 \ldots \ldots$ \\
\hline Low cervical: anterior aspect of intertransverse space of C5-7 & $10 \ldots$. & $11 \ldots \ldots$. \\
\hline 2nd rib: 2nd costochondral junction & $12 \ldots .$. & $13 \ldots \ldots .$. \\
\hline Lateral epicondyle: $2 \mathrm{~cm}$ distal to epicondyle & $14 \ldots \ldots \ldots$ & $15 \ldots \ldots .$. \\
\hline Dorsum $R$ forearm $\left({ }^{*}\right)$ : junction of proximal $2 / 3$ and distal $1 / 3$ & $16 \ldots \ldots * *^{*}$ & $*$ \\
\hline L thumbnail!*) & & $17 \ldots \ldots . . *$ \\
\hline SIDE & & \\
\hline $\begin{array}{l}\text { Greater trochanter: posterior to trochanteric prominence } \\
\text { SUPINE }\end{array}$ & $18 \ldots \ldots$. & $19 \ldots \ldots$. \\
\hline Knee: medial fat pad proximal to the joint line & 20. & 21. \\
\hline
\end{tabular}


of pain without grimace, flinch or withdrawal; 2 (moderate $)=$ pain plus grimace or flinch; 3 (severe) $=$ pain plus marked flinch or withdrawal; 4 (unbearable) = patient 'untouchable', withdraws without palpation. A point was considered tender if there was a spontaneous verbal affirmation of pain from the subject in response to firm pressure by the thumb-pad of examiner's dominant hand and with a scores greater than 0 4. In this way of standardized manual tender point survey system, 18 FMTP sites and 6 control sites were examined and positive FMTP scores were remarked in a body chart for each case and control subject.

\section{Measuring disease activity}

Systemic lupus erythematosus disease activity index (SLEDAI) (Bombardier et al., 1992) ${ }^{9}$ was used only for SLE patients as a good tool to see the disease activity. All nine organ systems which include 24 descriptors were asked or examined and the scores were put with pre assigned severity weights. The scores ranged from 0 (no disease activity) to 105 (maximum disease activity)

\section{The subject group classification}

SLE patients (cases) were classified for analysis into two subgroups, those who noted to have 10 or less FMTP were regarded as '0-10 FMTP' group and the rest who noted 11 or more FMTP were also considered as '> 11 FMTP group' The control subjects were also classified in two subgroups in the same way. All these activities have been done on the same day of scheduled visit to lupus clinic.

\section{Data Analysis}

\section{Editing raw data}

The predesigned data sheets were scrutinized 100 percent to check the quality of the raw data. It was basically a process of examination to detect errors, omission, and to correct these whenever possible. The hundred percent crosschecks were done after editing, which gave a good quality of data. $\mathrm{P}$ value 0.05 with $95 \%$ confidence interval was considered as the level of statistical significance.

\section{Use of computer}

After editing, the coded data were directly entered into the computer by using Statistical Package for Social Science (SPSS) 10.0 Windows Version. Analyses were performed using the software. This included descriptive statistics (mean, SD, Range, Percentage), Correlationcoefficient, Student's 't' test, chi-square test.

\section{Results}

A total 67 consecutive female systemic lupus erythematosus (SLE) patients and equal number of female healthy controls were enrolled in this observational study. Their characteristics, number of fibromyalgia tender points (FMTP) were studied in both groups and SLE disease activity index (SLEDAI) were studied in SLE group.

Sociodemographic characteristics: Sociodemographic characteristics of SLE patients and control subjects are shown in Table-I.

Five years interval was taken for age grouping in both groups. The age range was 16 to 55 years. The age (mean \pm SD) of the SLE patients and controls were $26.82 \pm 8.02$ and $29.67 \pm 10.80$ years respectively. No significant difference $(\mathrm{p}$ $=0.091)$ in age was observed in this study population. In this series, among the occupation 63\% was house-maker in SLE group. On the other hand student and service holder were $42 \%$ and $24 \%$ respectively in control group. The difference was statistically significant ( $p=0.004) .69 \%$ of control subjects were in $>X$ years educational level.48\% subjects in SLE group were in VI-X years educational level. Family income classes were nearly similar in both groups. No significant difference in family income $(p=0.065)$ was observed but significant difference was observed in level of education ( $\mathrm{p}=0.009$ ).

Baseline characteristics: In this series, the common clinical features were arthritis, oral ulcer, malar rash, photosensitivity and serositis; $86.6 \%, 82.1 \%, 67.2 \%$, and $10.4 \%$ respectively (Table-II). 
Table-I

Sociodemographic characteristics of systemic lupus erythematosus and control groups.

\begin{tabular}{|c|c|c|c|c|c|}
\hline \multirow[t]{2}{*}{ Parameter } & \multicolumn{2}{|c|}{ SLE $(\mathrm{n}=67)$} & \multicolumn{2}{|c|}{ Control $(\mathrm{n}=67)$} & \multirow[t]{2}{*}{$\mathrm{P}$} \\
\hline & No. & $(\%)$ & No. & $(\%)$ & \\
\hline \multicolumn{6}{|l|}{ Age (years) } \\
\hline $16-20$ & 19 & (28.4) & 22 & (32.8) & $0.091\left(\chi^{2}\right)$ \\
\hline $21-25$ & 15 & (22.4) & 8 & (11.9) & \\
\hline $26-30$ & 16 & (23.9) & 7 & $(10.4)$ & \\
\hline 31-35 & 9 & (13.4) & 13 & (19.4) & \\
\hline $36-40$ & 5 & (7.5) & 5 & $(7.5)$ & \\
\hline $41-45$ & 1 & (1.5) & 6 & (9.0) & \\
\hline $46-50$ & 1 & (1.5) & 4 & (6.0) & \\
\hline$>50$ & 1 & (1.5) & 2 & $(3.0)$ & \\
\hline Meanw \pm SD & \multicolumn{2}{|c|}{$26.82 \pm 8.02$} & \multicolumn{2}{|c|}{$29.67 \pm 10.80$} & 0.085 \\
\hline \multicolumn{5}{|l|}{ Occupation } & $0.004\left(\chi^{2}\right)$ \\
\hline House-maker & 42 & (62.7) & 23 & (34.3) & \\
\hline Service & 7 & (10.4) & 16 & (23.9) & \\
\hline Student & 18 & (26.9) & 28 & $(41.8)$ & \\
\hline \multicolumn{5}{|l|}{ Level of education } & $0.009\left(\chi^{2}\right)$ \\
\hline Cannot read/write & 0 & & 5 & (7.5) & \\
\hline Can read and sign & 0 & & 2 & (3.0) & \\
\hline I-V years & 10 & (14.9) & 4 & $(6.0)$ & \\
\hline VI-X years & 32 & (47.8) & 10 & (14.9) & \\
\hline > X years & 25 & $(37.3)$ & 46 & (68.7) & \\
\hline \multicolumn{5}{|c|}{ Family income (Taka/month) } & $0.065\left(\chi^{2}\right)$ \\
\hline $2,000-5,000$ & 22 & (32.8) & 21 & (31.3) & \\
\hline$>5,000-10,000$ & 31 & $(46.3)$ & 20 & $(29.9)$ & \\
\hline$>10,000-20,000$ & 12 & $(17.9)$ & 18 & $(26.9)$ & \\
\hline$>20,000$ & 2 & $(3.0)$ & 8 & $(11.9)$ & \\
\hline
\end{tabular}

Test: Chi-square test/unpaired Student's 't' test, SLE = Systemic Lupus Erythematosus $\mathrm{SD}=$ Standard Deviation

Table - II

Baseline characteristics of SLE patients at enrollment ( $n=67)$

\begin{tabular}{lcc}
\hline Character & No. & $(\%)$ \\
\hline Arthritis & 58 & $(86.6)$ \\
Oral/nasopharyngeal ulceration & 55 & $(82.1)$ \\
Malar rash & 45 & $(67.2)$ \\
Photosensitivity & 45 & $(67.2)$ \\
Discoid rash & 36 & $(53.7)$ \\
Serositis & 7 & $(10.4)$ \\
$\quad$ pleuritic pain/pleural rub/ pleural effusion/pericarditis & 61 & $(91.0)$ \\
Antinuclear antibody & 59 & $(88.1)$ \\
Immunologic disorder & & \\
$\quad$ anti-ds deoxyribo nucleic acid/anti-Smith antibody/ & \\
$\quad$ Rnti-ds deoxyribo nucleic acid/anti-Smith antibody/anti-phospholipid antibody/ & 23 & $(34.3)$ \\
$\quad$ proteinuria $>$ 0.05 gram/day cellular casts (RBC/WBC/tubular cell) & 22 & $(32.8)$ \\
Neurologic disorder & & \\
$\quad$ Psychosis/seizures & 14 & $(20.9)$ \\
$\quad$ Haemolytic disorder & & \\
$\quad$ haematologic anaemia/leucopenia lymphopenia/thrombocytopenia & &
\end{tabular}


The laboratory features of these patients were anti-nuclear antibody (ANA), Immunological disorder, renal disorder, neurological disorder and hematological disorders, $91 \%, 88.1 \%$, $34.3 \%, 32.8 \%$ and $20.9 \%$ respectively.

At enrollment FMTPs in both SLE and control groups and SLEDAI in SLE group were shown. The number of FMTP (mean \pm SD) of both the study groups was $8.80 \pm 6.40$ (SLE) and 3.63 \pm 4.90 (controls) respectively. The difference was statistically significant $(p<0.001)$ and the SLEDAI score (meanSD) of SLE Patients was $11.75 \pm 9.85$ as shown in Table- III.
Comparison of fibromyalgia tender points and disease activity: The SLE patients were subgrouped according to FMTP count into ' $\mathrm{O}-10$ FMTP' and '> 11 FMTP'groups. In Table -IV, the SLEDAI score (mean \pm SD) of 0-10 FMTP and > 11 FMTP group were $8.49 \pm 7.34$ and $17.58 \pm 11.17$ respectively, here statistically significant difference $(\mathrm{p}<0.001)$ was observed.

Correlates of fibromyalgia tender points and disease activity: In this SLE series, the disease activity SLEDAI was positively correlated $(\mathrm{r}=+0.439, \mathrm{p}$ $<0.001)$ (Table-V) with FMTPs in SLE patients.

Table- III

At enrollment FMTP in both groups and SLEDAI score in SLE group

\begin{tabular}{lccc}
\hline Parameter & $\begin{array}{r}\text { SLE }(\mathrm{n}=67) \\
(\text { Mean } \pm \text { SD })\end{array}$ & $\begin{array}{c}\text { Control }(\mathrm{n}=67) \\
(\text { Mean } \pm \text { SD) }\end{array}$ & $\mathrm{p}$ \\
\hline FMTP & $8.80 \pm 6.40$ & $3.63 \pm 4.90$ & $<0.001$ \\
Sledai score & $11.75 \pm 9.85$ & & \\
\hline
\end{tabular}

Test: Unpaired Student's ' $\mathrm{t}$ ' test

$\mathrm{SD}=$ Standard Deviation

SLE $\quad=$ Systemic lupus Erythematosus

FMTP $=$ Fibromyalgia Tender Point

SLEDAI = Systemic Lupus Erythematosus Disease Activity Index

Table IV

Association between FMTP and SLEDAI score in SLE patients ( $n=67)$

\begin{tabular}{lccc}
\hline Parameter & $\begin{array}{c}\text { SLE }(\mathrm{n}=67) \\
\text { FMTP 0-10 }(\mathrm{n}=43) \\
(\text { Mean } \pm \text { SD })\end{array}$ & $\begin{array}{c}\text { FMTP } 11(\mathrm{n}=24) \\
(\text { Mean } \pm \text { SD })\end{array}$ & $\mathrm{p}$ \\
\hline SLEDAI score & $8.49 \pm 7.34$ & $17.58 \pm 11.17$ & $<0.001$ \\
\hline
\end{tabular}

Test : Unpaired Student's 't' test

$\mathrm{SD}=$ Standard Deviation

SLE $\quad=$ Systemic lupus Erythematosus

FMTP $=$ Fibromyalgia Tender Point

SLEDAI = Systemic Lupus Erythematosus Disease Activity Index

Table-V

Correlation between SLEDAI score and FMTP count in $S L E(n=67)$

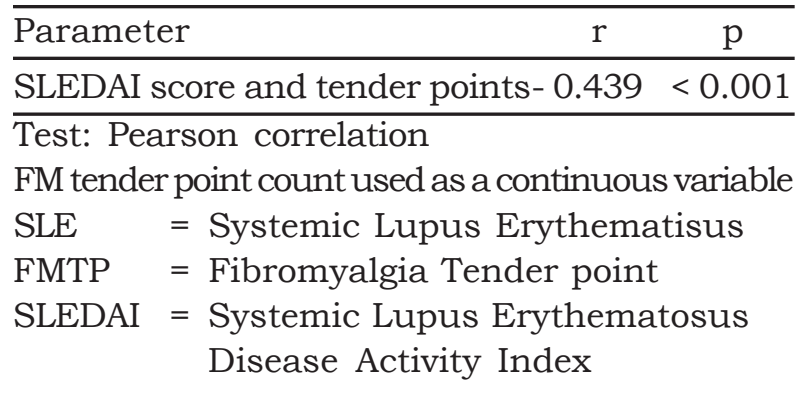

Disease duration: The disease duration (mean \pm SD) of all SLE patients was $42.03 \pm 37.90$ months (Table-VI). The $0-10$ and 11FMTP groups of SLE had disease duration (mean SD) of $43.84 \pm 39.99$ months and $38.79 \pm 35.66$ months respectively. The difference was not significant $(p=0.605)$ between these two groups.

Moreover, in this series no significant correlation was observed in SLEDAI $(r=+0.085$, $\mathrm{p}=0.495)$ with disease duration as shown in Table-VII. 
Table-VI

Total disease duration of SLE patients according to FMTP 0-10 and FMTP 11

\begin{tabular}{llrl}
\hline & \multicolumn{2}{l}{ Disease duration } & \\
\cline { 2 - 3 } Parameter & $\mathrm{n}$ & $\begin{array}{c}\text { Mean } \pm \mathrm{SD} \\
\text { (months) }\end{array}$ & $\mathrm{P}$ \\
\hline FMTP & & & \\
$0-10$ & 43 & $43.84 \pm 39.99$ & 0.605 \\
11 & 24 & $38.79 \pm 35.66$ & \\
\hline
\end{tabular}

Test: Unpaired Student's ' $t$ ' test

$\mathrm{SD}=$ Standard Deviation

SLE = Systemic Lupus Erythematosus

FMTP $=$ Fibromyalgia Tender Point

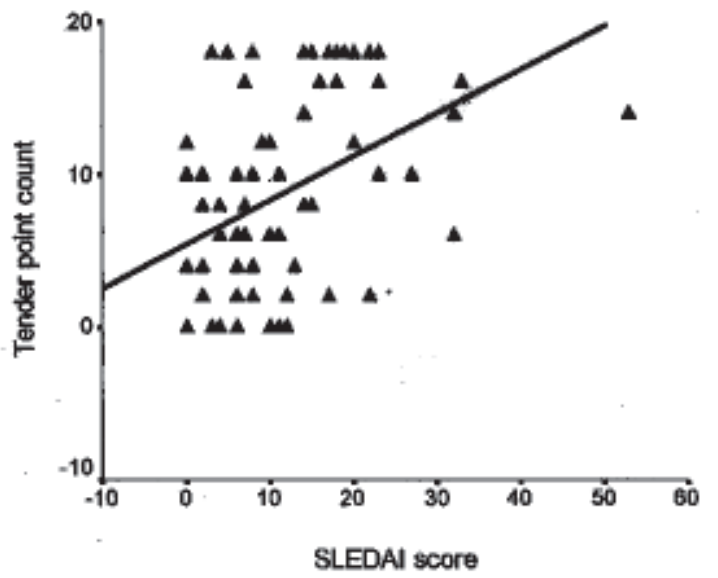

Fig.-2: Relationship between FMTP and SLEDAI in $S L E$

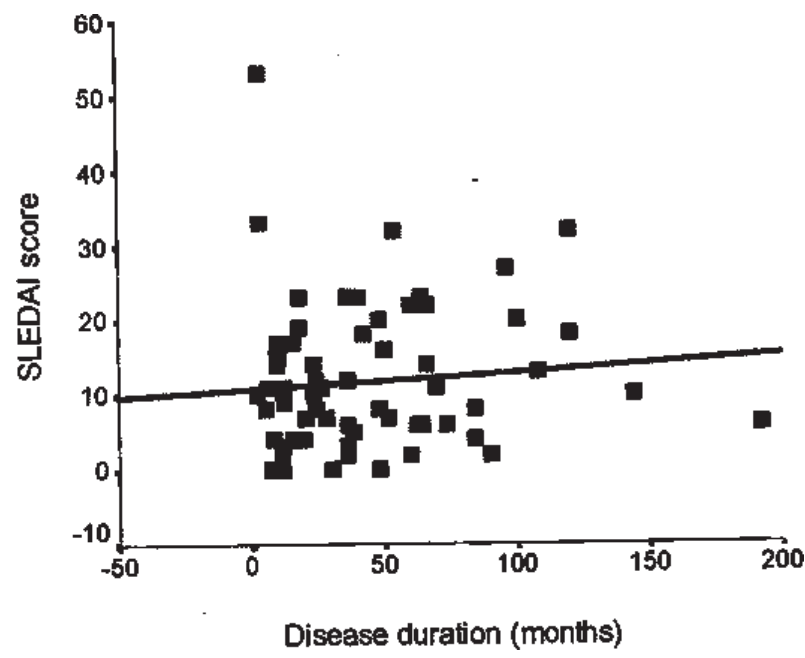

Fig.-3: Relationship between disease duration and SLEDAI in SLe
Table-VII

Correlation between disease duration and SLEDAI score in SLE $(n=67)$

\begin{tabular}{lcc}
\hline Parameter & $\mathrm{r}$ & $\mathrm{p}$ \\
\hline Disease duration vs & +0.085 & 0.495 \\
SLEDAI score & & \\
\hline
\end{tabular}

Test: Pearson correlation

$\mathrm{SD}=$ Standard Deviation

SLE = Systemic Lupus Erythematisus

FMTP = Fibromyalgia Tender point

SLEDAI = Systemic Lupus Erythematosus

Disease Activity Index

\section{Discussion}

The prevalence of fibromyalgia (FM) in general population is between $0.75 \%$ and $3 \%^{6}$. In Bangladesh its prevalence is $4.4 \%$ and $3.3 \%$ among rural and urban population respectively ${ }^{7}$. Coexistence of FM and SLE was reported in $22 \%$ and $25 \%$ in the studies from North America and Australia respectively. However, a lower percentage was reported from India $(8.2 \%)$ and Spain $(10 \%)^{14,15}$.

Da Costa et al. (2000) ${ }^{11}$ examined 46 FM and 59 SLE patients. The mean ages were 48.3 and 42.36 years in FM and SLE patients respectively. Handa et al. (1998), ${ }^{5}$ in their series of 158 SLE patients, observed near similar mean ages in FM and without FM group (37.8 \pm 13.98 vs $34.06 \pm 11.36$ years). In total $189 \mathrm{SLE}$ women were classified into FM, regional pain (RP) and no pain (NP) group in the study of Valencia-Flores et al. (2004) ${ }^{15}$. They observed nearly similar mean ages among the 3 subgroups $(37.8 \pm 11.3,39 \pm 13.4$ and 39.8 years respectively). In our series, age and sex matched control were enrolled. The age range was $16-55$ years. The mean age was $26.82 \pm 8.02$ and $29.67 \pm 10.86$ years respectively of SLE and control population. Moreover, the mean ages of both patients and control subjects were observed lower in comparison to the others. This observation could be due to lesser number of aged patients attended in the SLE clinic. It could also be the reflection of both the SLE disease and fibromyalgia that might occur in early part of life. As prevale nce study of rheumatologic diseases in Bangladesh observed the mean age $32.8 \pm 15.6,30.7 \pm 12.4$ and $32.3 \pm 14.6$ years in rural, urban and urban affluent respectively ${ }^{7}$. 
Occupation distributions in our series were house-maker, service and student with frequency of 63, 10 and 27\% in SLE patients. In control, these occupations were 34, 24 and $42 \%$ respectively. Da Costa et al. $(2000)^{11}$, in their study observed $48 \%(n=22)$ of patients with FM were working full or part time compared to $49 \%(n=29)$ women with SLE.

Household income was significantly higher for FM group compared to SLE group observed in the study of Da Costa et al. (2000) ${ }^{11}$. In this study, family income classes were observed similar in both SLE and control groups. No significant difference was revealed in family income $(p=0.065)$.

Frequency of musculoskeletal and mucocutaneous features were observed more than $92 \%$ ( $n=148$ SLE) by Handa et al. $(1998)^{5}$ in their series. Arthritis was $>70 \%$ (mean $85 \%)$ and higher percentage of antinuclear antibody (98\% and 96\%) reported by Kumar $(2000)^{16}$ in their northern and southern Indian study population respectively. Morand et al. $(1994)^{14}$ in their series, observed higher frequency in arthralgia / arthritis (71\%). In our series, arthritis and antinuclear antibody were $86.6 \%$ and $91 \%$ respectively.

Handa et al. (1998) ${ }^{5}$ reported that lupus patient with FM had higher number of tender points (14.23 \pm 1.13$)$ compared to non FM patients $(1.56 \pm 1.77)$. None of the control subjects exhibited any tender points. Their study results were also partially consistent with the results of this study, where SLE group showed tender points (8.80 .4) and control subjects (3.63 \pm 4.9$)$.

Although SLEDAI has a theoretical possible score of up to 105 , which means 9 organ systems and 24 descriptors present simultaneously, an impossible event ${ }^{9}$. Score ranges from 0 (no disease activity) to 105 (maximum disease activity). A score of 6 is considered clinically important and influences the majority of physicians to treat the patients (Da Costa et al., (2000) ${ }^{11}$. However, in our study $(n=67)$, score range of SLEDAI was 5 - 25, though one patient scored above 50 .

Middleton et al. (1994) ${ }^{6}$, in their series, observed that the SLEDAI was correlated with
FMTP count but Gladman et al. $(1997)^{12}$, in their study, found that FM as assessed by the tender point count, did not correlate to the total score or to any of the component of SLEDAI but correlated with all domains of SF-36. They also reported that, in clinical practice and trails, the presence of FMTP may musk the effect of treatment on disease activity. As well, when SLEDAI descriptors were grouped into 9 organ systems, no relationship was also found ${ }^{8}$. However, Akkasilpa et al. (2005) ${ }^{2}$ found association of arthritis descriptor of SLEDAI with FMTP. In our study, we observed that SLEDAI was significantly $(p<0.001)$ correlated $(\mathrm{r}=+0.439)$ with FMTP count i.e. more SLEDAI score in $>11$ FMTP group. This result supported the observations of Middleton et al. (1994) ${ }^{6}$ and Akkasilpa et al. (2005) ${ }^{2}$.

Handa et al. $(1998)^{5}$, in their series, observed no correlation between disease duration and SLEDAI. Akkasilpa et al. (2005) ${ }^{2}$, in their study, found no correlation between disease duration and FMTP. However, in our study, the disease duration in SLE patients (Mean SD) was 42.03 37.90 months. No significant association or correlation of disease duration with FMTP count $(\mathrm{p}=0.605)$ and SLEDAI score $(\mathrm{r} \mathrm{a}=+0.085$, p 0.495) was observed. This result supported the observations of Handa et al. (1998) ${ }^{5}$ and Akkasilpa et al. (2005) ${ }^{2}$. So, disease duration does not have influence on tender points and disease activity.

These studies do provide a likely indication of fluctuation of number of FMTP and SLEDAI score in the natural course of SLE. So patients should be followed up over time to determine how FM symptoms vary in conjunction with SLE disease activity. Moreover, prospective outcome and disease activity will ultimately be required to assess prognostic importance of concomitant FMTP in SLE.

Age matching of the study subjects made the data uniform. Subgrouping of SLE patients clearly expressed the association of FMTPs with disease activity. It may be stated that FMTPs were found more in number in those who had increased SLEDAI score.

Furthermore, it was exhibited that presence of FMTP may confuse the disease activity due to 
common overlapping symptoms. SLE patients with > 11 FMTP count are likely to have an important negative impact on disease activity. However, FMTP count may not only be empirically helpful in the evaluation of disease activity.

\section{Limitations}

In this study, the sample size was small and another limitation was that dolorimeter had not been used. So that, approximate force of 4 $\mathrm{kg} / \mathrm{cm}^{2}$ couldn't been applied rather pressure by thumbs which whiten the nail beds had been applied Masking effect of FM on treatment outcome could not be observed due to lack of follow up.

\section{Conclusion:}

SLE patients with higher number of Fibromyalgia tender points are significantly associated with increased score of disease activity. The more FMTP count is found the more correlation is observed with SLEDAI score in SLE patients. Disease duration does not have influence on tender points and disease activity.

Even though, the evaluation of disease activity should not be emphasized by counting FMTP only, because fibromyalgia is not an inflammatory disease. Since FM has masking effect on the treatment outcome of SLE, during management individualization of patients is recommended. Their FM should be addressed separately when necessary. Further studies will need to observe the variation of FM symptoms in relation to disease activity. Such information could have important implications for the interpretation of patients' symptoms and in the treatment plan in the setting of SLE.

\section{Acknowledgments:}

The authors wish to acknowledge Professor Syed Atiqul Haq, Department of Rheumatology, BSMMU, Dhaka, and Professor MA Jalil Chowdhury, Department of Medicine, BSMMU, Dhaka, for their help and over all guidance for the study.

\section{References}

1 Taylor J, Skan J, Erb N, Carruthers D, Bowman $\mathrm{S}$, Gordon C, Isenberg D. Lupus patients with fatigue-is there a link with fibromyalgia sydrome? Rheumatology 2000; 39: 620-3.

2 Akkasilpa S, Goldman D, Magder LS, Petri M. Number of fibromyalgia tender points is associated with health status in patients with systemic lupus erythematosus. J Rheumatol 2005; 32: 48-50.
3 Bennett $\mathrm{R}$. The scientific basis for understanding pain in fibromyalgia. Downloaded from email archive of Dream Pharm.com. 2003.

4 Wolfe F, Smythe HA, Yunus MB, Bennett RM, Bombardier C, Goldenberg DL. The American College of Rheumatology 1990 criteria for the classification of fibromyalgia: report of the multicenter Criteria Committee. Arth Rheumat, 1990; 33: 160-72.

5 Handa R, Aggarwal P, Wali JP, Wig N, Dwivedi SN. Fibromyalgia in Indian patients with SLE. Lupus 1998; 7: 475-8.

6 Middleton GD, McFarlin JE, Lipsky PE. The prevalence and clinical impact of fibromyalgia in systemic lupus erythematosus. Arth Rheumatol 1994; 37: 1181-8.

7 Haq SA, Darmawan J, Islam MN, Uddin MZ, Das BB, Rehman F. Prevalence of rheumatic diseases and associated outcomes in rural and urban communities in Bangladesh: a COPCORD study. J Rheumatol 2005; 32: 348-53.

8 Haq I. Isenberg DA. How does one assess and monitor patients with systemic lupus erythematosus in daily clinical practice? Best Practice Res Clin Rheumatol 2002; 16: 181-94.

9. Bombardier C, Gladman DD, Urowitz MB, Caron $\mathrm{D}$, Chang $\mathrm{CH}$, Committee on prognosis in SLE. Derivation of the SLEDAI, A disease activity index for lupus patients. Arth Rheumatol 1992; 35: 630-40.

10 Hay E, Gordon C, Emery P. Assessment of lupus: where are we now? Ann Rheum Dis 1993; 52: 169-72.

11 Da Costa D, Dobkin PL, Fitzcharles MA, Fortin PR, Beaulieu A, Zummer M. Determinants of health status in fibromyalgia: a comparative study with systemic lupus erythematosus. J Rheumatol 2000; 27: 365-72.

12 Gladman DD. Urowitz MB. Ong A. Gouch J MacKinno n A. Fibromyalgia is a major contributor to quality of life in lupus. J Rheumatol 1997; 24: 2145-8.

13 Okufuji A, Turk DC, Sinclair JD, Starz TW, Marcus DA. A standardized manual tender point survey. I. Development and determination of a threshold point for the identification of positive tender points in fibromyalgia syndrome. J Rheumatol 1997; 24: 377-83.

14. Morand EF, Miller MH, Whittin S, Littlejohn GO. Fibromyalgia syndrome and disease activity in systemic lupus erythematosus. Lupus 1994; 3: 187-91.

15. Valencia-Flores M, Cardiel MH, Santiago V, Resenidz M, Castano VA, Negrete O. Prevalence and factors associated with fibromyalgia in Mexican patients with systemic lupus erythematosus. Lupus 2004; 14: 4-10.

16. Kumar A. Indian guidelines on the management of SLE. J Indian Rheumatol Assoc 2000; 10: 80-96. 